\title{
Scope of Polymer Nano-composite in Bio-medical Applications
}

\author{
Kiran Kumar kudumula \\ (Mechanical Engineering Department, Sri Satya Sai University of Technology and Medical Sciences, India)
}

\begin{abstract}
The efforts are geared to the development and application of environmentally friendly and sustainable bio-reinforced composites for use in automotive, construction, packaging and medical fields. In the study of Green polymer nanocomposites we learned unique properties of combining the advantages of natural fillers and organic polymers. Plant fibers are found suitable to reinforce polymer. They are also biodegradable and are annually renewable compared to other fibrous materials. In addition to presenting the scientific framework for the advances in polymer nanocomposite research, it focuses on the scientific principles and mechanisms in relation to the methods of processing and manufacturing with a discussion on commercial applications and health/safety concerns.
\end{abstract}

Keywords: microscopy, polymers, composites, quantitative, statistical etc.

\section{Introduction}

In the last 20 years, there has been a strong emphasis on the development of polymeric nanocomposites, where at least one of the dimensions of the filler material is of the order of a nanometer. The final product does not have to be in nanoscale, but can be micro- or macroscopic in size. This surge in the field of nanotechnology has been greatly facilitated by the advent of scanning tunneling microscopy and scanning probe microscopy in the early 1980s. With these powerful tools, scientists are able to see the nature of the surface structure with atomic resolution. That addition of nanoparticles to base polymers confers improved properties that make them usable in automotive, construction and medical areas. Properties which have been shown to improve substantially are mechanical properties (e.g., strength, elastic modulus and dimensional stability), thermo mechanical properties and permeability (e.g., gases, water and hydrocarbons). Polymer nanocomposites are new class of particle filled polymers for which at least one dimension of the dispersed particles is in the nanometres range. Nanocomposite materials consisting of polymeric matrix and nano-scale particles have offered a great opportunity in thermoplastic and rubber industry to make new products/applications with enhanced/unique properties. Although applications of polymeric nanocomposites grow significantly due to the potential capability of addressing current challenges faced in the polymer industry, the potential impact of polymeric nanocomposites on environment should be considered. Nanocomposite is a class of composites in which the dimensions of the reinforcing material are in the order of nanometers. Because of this nanometres size characteristic, nanocomposites possess superior properties than the conventional composites due to maximizing the interfacial adhesion.

An improvement in a property arises when the length scale of the morphology (i.e., nano) and fundamental physics associated with a property coincide. Two principal factors cause the properties of nanomaterials to differ significantly from other materials: increased relative surface area and quantum effects. Some nanocomposites may show properties predominated by the interfacial interactions and others may exhibit the quantum effects associated with nanodimensional structures. For manufacturing of nano-phased structural polymer composite material, the first step will be choice of a fabrication method. Some of the widely used methods for manufacturing conventional composite parts are wet lay-up, pultrusion, resin transfer molding (RTM), vacuum assisted resin transfer molding (VARTM), autoclave processing, resin film infusion (RFI), prepreg method, filament winding, fiber placement technology, etc.

The field of nanotechnology is one of the most popular areas for current research and development in basically all technical disciplines. This obviously includes polymer science and technology and even in this field the investigations cover a broad range of topics. This would include microelectronics (which could now be referred to as nanoelectronics) as the critical dimension scale for modern devices is now below $100 \mathrm{~nm}$. Other areas include polymer-based biomaterials, nanoparticle drug delivery, miniemulsion particles, fuel cell electrode polymer bound catalysts, layer-by-layer self-assembled polymer films, electrospun nanofibers, imprint lithography, polymer blends and nanocomposites. Even in the field of nanocomposites, many diverse topics exist including composite reinforcement, barrier properties, flame resistance, electro-optical properties, cosmetic applications, bactericidal properties. Nanotechnology is not new to polymer science as prior studies before the age of nanotechnology involved nanoscale dimensions but were not specifically referred to as nanotechnology until recently. Phase separated polymer blends often achieve nanoscale phase dimensions; block copolymer domain morphology is usually at the nanoscale level; asymmetric membranes often have nanoscale void 
structure, miniemulsion particles are below $100 \mathrm{~nm}$; and interfacial phenomena in blends and composites involve nanoscale dimensions.

Thermal fluctuations in BCP materials characteristically drive the ordering phase transition order from second to first-order by the well known Brazovskii mechanism and there have been many observations of jumps in X-ray and neutron scattering intensity data at the order-disorder transition (ODT) that signal this phenomenon. However, the existence of quenched disorder associated with extensive nanoparticle clustering can be expected to destroy the ODT if their perturbing effect on the ordering process becomes sufficiently large. Yue et al. have found this effect in the dispersion of $\mathrm{C}_{60}$ into a model (polystyrene-block-polyisoprene) $\mathrm{BCP}$ material. In particular, small angle X-ray scattering data indicated that a small amount (1 mass \%) of $\mathrm{C}_{60}$ caused the $\mathrm{BCP}$ to remain disordered over a wide temperature range so that a $\mathrm{BCP}$ ordering phase transition no longer existed. This phenomenon offers both technological problems and opportunities.

\section{Literature Review}

RTO Lecture Series et.al (2005) In the last 20 years, there has been a strong emphasis on the development of polymeric nanocomposites, where at least one of the dimensions of the filler material is of the order of a nanometer. The final product does not have to be in nanoscale, but can be microor macroscopic in size.

Pandey, J.K et.al (2007) The use of polymer composites from renewable sources has advantages over synthetic sources, particularly as a solution to the environmental problems generated by plastic waste.

Zou, H. et.al (2008) The one step in-situ polymerization method involves the dispersion of nanofillers in monomer(s), followed by bulk or solution polymerization. The nanofillers are always modified by functional groups to increase the interaction between the polymers and the nanofillers, or to get a good dispersion in the polymer matrix. This method has been reported to have many advantages, such as ease of handling and better performance of the final products.

Tang et al (2008) have reported that inclusion of dry powder $\mathrm{SiO}_{2}$ particles in starch $\mathrm{PVOH}$ films increased tensile strength at break and improved water barrier properties.

Xiong et al (2008) have reported improved mechanical properties, transmittance, and water resistance of starch films containing nano-SiO2 particles.

\subsection{Noteworthy Contributions}

Sinha, S.R et.al (2011) (They offer alternatives to maintaining sustainable development of economic and ecologically attractive technology.

Majdzadeh-Ardakani et.al (2010) Although the effect of the method on the properties of the resulting composite has not been reported, it is believed that the process method leads to better exfoliation of fibers in the matrix. Melt extrusion is another method frequently been used.

Mazumder, S.K. (ed.). (2012) Some of the widely used methods for manufacturing conventional composite parts are wet lay-up, pultrusion, resin transfer molding (RTM), vacuum assisted resin transfer molding (VARTM), autoclave processing, resin film infusion (RFI), prepreg method, filament winding, fiber placement technology, etc.

Kaplan DL, Mayer JM, Ball D, McCassie J, Allen AL, Stenhouse P (2011) A vast number of biodegradable polymers (e.g. cellulose, chitin, starch, polyhydroxyalkanoates, polylactide, polycaprolactone, collagen and other polypeptides...) have been synthesized or are formed in natural environment during the growth cycles of 2 Biodegradable Polymers 15 organisms. Some micro organisms and enzymes capable of degrading such polymers have been identified.

Biodegradable Plastics et.al (2012) Thermoplastic starches are beginning to show increased commercial use. There are many challenges still to be overcome in order to make commercially viable biodegradable and compostable starch polymers. Thermoplastic starch films generally need to have starch content greater than $70 \%$ to have biodegradable or compostable action.

Billmeyer, F.W.J et.al (2010) The term composite refers to matrix polymers that contain dispersed conventional filler, such as glass, fibres, talc or clay particles. Polymers with fillers that are nano-sized in scale are named nano-composites. The properties of a composite depend on the volume fraction of the filler, its shape, size and interfacial adhesion. 


\section{Outcome of the study}

Upon making an enumerative literature survey in Fig.1 illustrates us that the majority of the applications on nano composites is in automotive and aerospace sector. In intermediate range they are used in sports and domestic appliances. But in Bio applications and in medical filed very less emphasis is being made, so it gives a propulsion of lot to be done in this field of research.

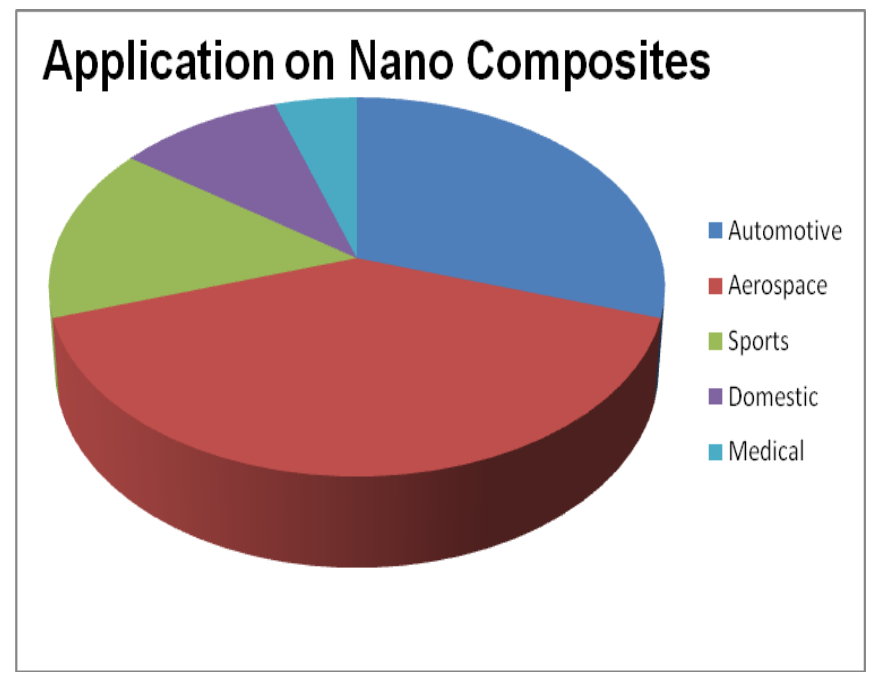

Figure 1Application of Nano composites in different fields

In fig. 2 it can be visualized that most of the researchers are working in synthesis of nano composites. As a point of view of contributing from engineering perspective lot needs to worked on in the fields of testing, simulation, characterization and modeling of polymer nano composites.

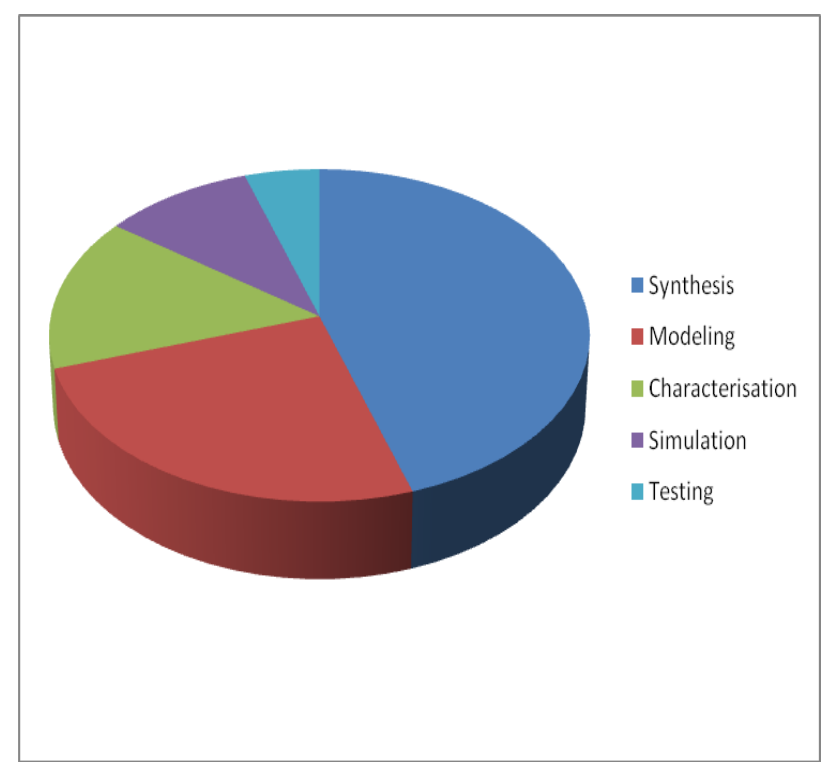

Figure 2 Researchers working in the broad area of Nano Composites

\section{Research Methodology}

Descriptive research is also called Statistical Research. The main goal of this type of research is to describe the data and characteristics about what is being studied. The idea behind this type of research is to study frequencies, averages, and other statistical calculations.

It is quantitative and uses surveys and panels and also the use of probability sampling. Descriptive research is the exploration of the existing certain phenomena. The details of the facts won't be known. The existing phenomena's facts are not known to the persons.

As of now in this paper descriptive research is being carried out and upon completing problem identification stage the research is progressed towards quantitative and qualitative techniques. 


\section{Conclusion}

This study motivates that there is deficiency of research is biomechanics field and necessity in investigating the mechanical characteristics of polymer nano-composites and its applications in the field of bio-mechanics and discuss the various issues related to it.

\section{Journal Papers:}

\section{References}

[1]. Majdzadeh-Ardakani, K.; Sadeghi-Ardakani, Sh., "Experimental investigation of mechanical properties of Starch/natural rubber/clay nanocomposites", Digest J. Nanomater. Biostruct. 2010, 5, 307-316.

[2]. Mazumder, S.K. (ed.). (2012), “Composites Manufacturing, Materials, Product \& Process Engineering”, CRC Taylor \& Francis, ISBN0-8493-0585-3.

[3]. Kaplan DL, Mayer JM, Ball D, McCassie J, Allen AL, Stenhouse P (2011), "Fundamentals of Bio-degradable polymers", In: Ching C, Kaplan DL, Thomas EL (eds) Biodegradable polymers and packaging. Technomic Pub Co, Lancaster, pp 1-42.

[4]. Tang S., Z., P., Xiong, H., Tang, H., "Effect of nano-SiO2 on the performance of starch/polyvinyl alcohol blend films. Carbohydrate Polymers", 2008. 72: p. 521-526.

[5]. Billmeyer, F.W.J., "Textbook of Polymer Science”. 3 ed. 2010: John Wiley \& Sons. Inc RTO Lecture Series, EN-AVT-129, May 2005.

\section{Chapters in Books:}

[1]. Sinha, S.R.; Bousmina, M. Biodegradable polymer/layered silicate nanocomposites. In Polymer Nanocomposites Mai, Y., Yu, Z., Eds.; Wood head Publishing and Maney Publishing: Cambridge, England, pp. 57-129.

[2]. Xiong H., T.S., Tang, H., Zou, P. The structure and properties of a starch-based biodegradable film. Carbohydrate Polymers 2008(263-268).

[3]. Biodegradable Plastics - Developments and Environmental Impacts E. Australia, Editor. 2012, Nolan Itu Pty Ltd.

[4]. Zou, H.; Wu, S.S.; Shen, J. Polymer/silica nanocomposites: preparation, characterization, properties, and applications Chem. Rev, $2008,108,3893-3957$

\section{Proceedings Papers:}

[1]. $\quad$ Pandey, J.K.; Chu, W.S.; Lee, C.S.; Ahn, S.H., "Preparation characterization and performance evaluation of nanocomposites from natural fiber reinforced biodegradable polymer matrix for automotive applications", Presented at the International Symposium on Polymers and the Environment: Emerging Technology and Science, Bioenvironmental Polymer Society (BEPS), Vancouver, WA, USA, 17-20 October 2007. 